\title{
Adaptive Model Based Pulmonary Artery Segmentation in 3-D Chest CT
}

\author{
Marco Feuerstein $^{a}$, Takayuki Kitasaka $^{b, c}$, Kensaku Mori ${ }^{a, c}$ \\ ${ }^{a}$ Graduate School of Information Science, Nagoya University, Japan; \\ ${ }^{b}$ Faculty of Information Science, Aichi Institute of Technology, Japan; \\ ${ }^{c}$ MEXT Innovation Center for Preventive Medical Engineering, Nagoya University, Japan
}

\begin{abstract}
The extraction and analysis of the pulmonary artery in computed tomography (CT) of the chest can be an important, but time-consuming step for the diagnosis and treatment of lung disease, in particular in non-contrast data, where the pulmonary artery has low contrast and frequently merges with adjacent tissue of similar intensity. We here present a new method for the automatic segmentation of the pulmonary artery based on an adaptive model, Hough and Euclidean distance transforms, and spline fitting, which works equally well on non-contrast and contrast enhanced data. An evaluation on 40 patient data sets and a comparison to manual segmentations in terms of Jaccard index, sensitivity, specificity, and minimum mean distance shows its overall robustness.
\end{abstract}

Keywords: Segmentation, Vascular Image Processing, Shape

\section{INTRODUCTION}

Computed tomography (CT) of the chest is a very common means of diagnosing lung disease and related illnesses. As diagnosis is usually performed manually in high-resolution data, it can be very time-consuming and laborious. To aid the physician during this process, we are currently developing a segmentation pipeline for fully automatic extraction of the major vasculature and tracheobronchial tree inside the mediastinum. This will aid the physician in the automated detection of mediastinal, aortic, and N1 lymph nodes and in the assignment of lymph node stations, ${ }^{1}$ which in turn can guide bronchoscopy or biopsy. ${ }^{2}$

Since diagnosis can be performed in both contrast enhanced and non-contrast $\mathrm{CT},{ }^{3}$ our method needs to handle both types equally well. It also needs to properly deal with imaging artifacts due to cardiac motion or calcifications and adjacent (seemingly merging) vasculature, which is in particular difficult for non-contrast CT.

Having finished the first two steps of automatic tracheobronchial tree ${ }^{4}$ and aortic arch segmentation, ${ }^{5}$ we here present the next step in our segmentation pipeline: automatic extraction of the pulmonary artery, i.e. pulmonary trunk and left and right pulmonary artery.

Further author information: (Send correspondence to M.F.)

M.F.: E-mail: fmarco@suenaga.m.is.nagoya-u.ac.jp, Telephone: +81 527895688

T.K.: E-mail: kitasaka@aitech.ac.jp

K.M.: E-mail: kensaku@is.nagoya-u.ac.jp

Copyright 2010 Society of Photo-Optical Instrumentation Engineers.

This paper was published in SPIE Medical Imaging and is made available as an electronic reprint with permission of SPIE. One print or electronic copy may be made for personal use only. Systematic or multiple reproduction, distribution to multiple locations via electronic or other means, duplication of any material in this paper for a fee or for commercial purposes, or modification of the content of the paper are prohibited. 


\section{RELATED WORK}

Up to now, many previous works concentrated primarily on the extraction of pulmonary vessel trees inside the lung, ${ }^{6-10}$ but without extracting the main branches (pulmonary trunk, left and right pulmonary artery), as they often merge with adjacent tissue. They also mainly work with contrast enhanced data, which is in general easier to process than non-contrast CT.

Only a few approaches were presented that extract the pulmonary artery. ${ }^{2,11-14}$ They either only work on contrast enhanced data, ${ }^{12-14}$ have been tested on a very limited set of cases only, ${ }^{11-13}$ require user interaction (e.g. five to eight user selected landmarks ${ }^{12}$ or a manually placed seed point ${ }^{13}$ ), or fail for up to $20 \%$ of cases, ${ }^{2,11}$ as the utilized fixed model or models are not flexible enough. We here present the first work that addresses these problems: Using an adaptive model, it works equally well on a large number of contrast enhanced and non-contrast data sets.

\section{METHOD}

Our method, which is an extension and improvement of previous works on pulmonary artery extraction, ${ }^{2,11}$ first generates a rough patient-specific centerline of the pulmonary artery based on an adaptive anatomical model and circular Hough transforms. After approximating this centerline with nonuniform rational B-spline (NURBS) curves, we fit the splines to a likelihood image consisting of Euclidean distances to possible pulmonary artery edges. As the pulmonary artery is of approximate tubular shape, the higher the Euclidean distance the higher the likelihood of a voxel to be part of the centerline of the pulmonary artery. After fitting, the full pulmonary artery lumen is recovered by a reverse Euclidean distance transform.

In contrast to previous work, ${ }^{2,11,15}$ which uses one or more fixed centerline models that cannot be successfully fitted to the highly individual anatomy of every patient, we here present an adaptive model that can be fitted to the patient-specific anatomy. This also makes the subsequent B-spline fitting less complex and hence more robust.

\subsection{Preprocessing}

In the remainder of the paper, we assume the patient to be placed in supine position, as this is the case for most chest CT acquisitions. However, if the patient was positioned differently, we could identify unique representative anatomic landmarks such as bones, ${ }^{16}$ estimate the main body axes from these landmarks, and rotate the data accordingly.

Furthermore, our method utilizes a segmentation of the aortic arch. It can be obtained automatically e.g. by our previously developed method, ${ }^{5}$ which works robust on contrast enhanced as well as non-contrast CT data. This aortic arch segmentation serves two purposes. First, we can utilize the arch as important anatomical landmark, since the right pulmonary artery passes it orthogonally and the left pulmonary artery runs along it (see Fig. 4). Second, we can mask out all arch voxels for further processing, which is important during circular Hough transform in 2-D slices containing circular cross sections of both the aorta and the pulmonary artery (cf. Sec. 3.2.1).

\subsection{Pulmonary Artery Extraction}

Our pulmonary artery extraction is a two-step approach. In the first step, we generate a patient-specific approximate centerline of the pulmonary artery from our adaptive model (see Sec. 3.2.1). In the second step, we match this centerline to the actual centerline and recover the full lumen of the artery (see Sec. 3.2.2).

\subsubsection{Adaptive Model Based Hough Transform}

Our adaptive pulmonary artery model is built relative to a semicircle approximating the aortic arch (see Fig. 1). The centerline of the pulmonary artery is constructed from the centers of circles resulting from a series of circular Hough transforms inside oblique square 2-D slices. We name these oblique 2-D slices either key or model slices. Key slices determine the overall size and orientation of the model and hence adapt the model to the patient, while model slices are centered along predefined paths relative to the aortic arch. 


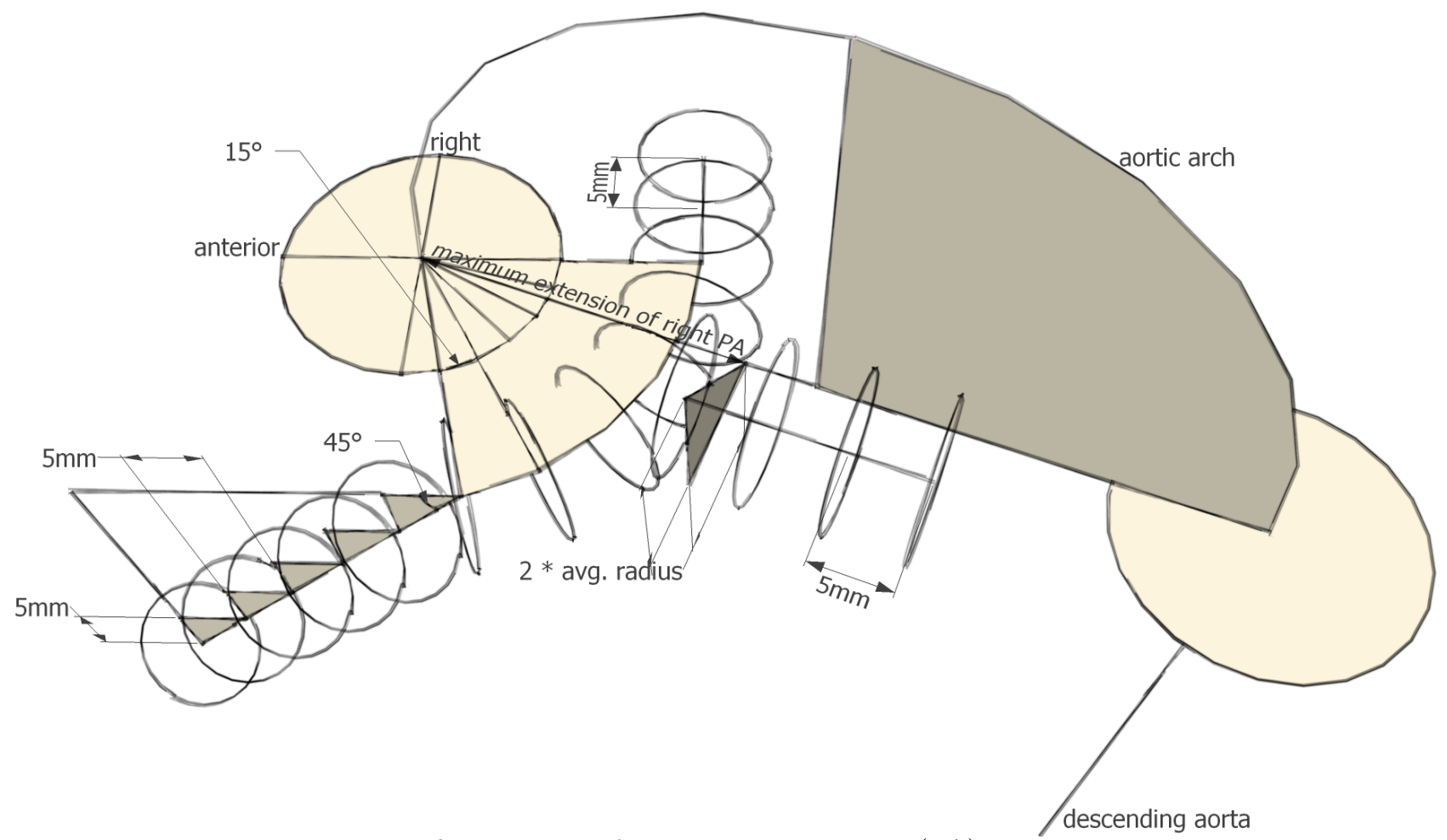

Figure 1: Patient-specific modeling of the pulmonary artery (PA) relative to the aortic arch

The first key slice is the plane enclosing the semicircle of the aortic arch. As the pulmonary artery orthogonally cuts this plane, we can easily estimate a first centerline point by searching for Hough circles inside or below the semicircle. In the axial slice containing this centerline point, we now compute the distance of this centerline point to the centerline of the ascending aorta and take it as an estimate for the radius of the curve of the right pulmonary artery that runs around the ascending aorta. We utilize this curve radius to search for six more centerline points, generating oblique (model) slices $15^{\circ}$ away from each other and starting at the model slice enclosed by the sagittal plane going through the center of the ascending aorta. We consider the centerline point detected in the model slice $75^{\circ}$ away from the sagittal slice to be our initial branching point where the pulmonary trunk bifurcates into the left and right artery. Descending from this branching point, we estimate the pulmonary trunk by five more model slices oriented $45^{\circ}$ towards axial and coronal slices and spaced $5 \mathrm{~mm}$ each along the superior-inferior and anteroposterior axis. To the right of the sagittal slice going through the center of the ascending aorta, two more centerline points are approximated by model slices spaced $5 \mathrm{~mm}$ and parallel to the sagittal planes.

After estimating the pulmonary trunk and the right pulmonary artery, we generate a second key slice, which is orthogonal to the plane enclosing the semicircle of the aortic arch. It is placed the maximum extension of the right pulmonary artery away from the ascending aorta, i.e. the maximum radius of the right pulmonary artery plus the maximum curve radius, and is shifted two times the average pulmonary artery radius to the superior and to the left (orthogonal to the plane enclosing the semicircle of the aortic arch). Three more 5mm-spaced model slices are generated parallel to this key slice towards the descending aorta. Last, we generate four equally spaced oblique model slices in between the centerline point found in the second key slice and the initial branching point (not shown in Fig. 1).

In order to avoid outliers during circular Hough transform, we restrict the size of all model slices to be four times the average radius of the respective pulmonary artery branch (trunk, left, right), which is iteratively recomputed. The size of the first key slice enclosing the semicircle of the aortic arch is four times the radius of the semicircle, while the size of the second key slice orthogonal to the first key slice is six times the average radius of the pulmonary artery. Furthermore, not altering the main directions of the adaptive model, we align 
the new center of a reconstructed slice with the circle center found in the previous slice, so we can deal with different bending and curvature conditions (not shown in Fig. 1).

During Hough circle extraction, the Hough map is computed only considering pixels between $t_{\mathrm{H}_{\min }}$ and $t_{\mathrm{H}_{\max }}$ in the input image, which is the typical edge intensity range of vessels. After the first Hough transform performed in the first key slice, the upper threshold $t_{\mathrm{H}_{\max }}$ is automatically adjusted to the intensity of the Hough circle center found in the first key slice to account for contrasted data, where the range of edge voxels is much larger. For each pixel, we compute the Gaussian derivative of standard deviation $\sigma_{\mathrm{H}}$ to estimate the direction of the normal at that pixel. The Hough accumulator is filled by drawing lines between $r_{\mathrm{H}_{\min }}$ and $r_{\mathrm{H}_{\max }}$ (the typical minimum and maximum radii of the pulmonary artery) along the normal. We blur the Hough map using a variance of $v_{\mathrm{H}}$ and compute the maxima in the Hough map.

In contrast to our previous work on segmentation of the aortic arch, ${ }^{5}$ which is the thickest vessel in the mediastinum, the pulmonary artery can have a radius similar to other vasculature. To select the correct circle representing the pulmonary artery from the maxima found in the Hough map, we ignore all voxels previously marked as aortic arch during Hough transform and perform following voting to distinguish the pulmonary artery from other vessels after Hough transform:

$$
p=\underset{i=1 \ldots n}{\arg \max }\left(\frac{h\left(\boldsymbol{x}_{i}\right)}{\max _{i=1 \ldots n}\left(h\left(\boldsymbol{x}_{i}\right)\right)} \cdot \frac{r\left(\boldsymbol{x}_{i}\right)}{\max _{i=1 \ldots n}\left(r\left(\boldsymbol{x}_{i}\right)\right)} \cdot \frac{d_{\mathrm{cen}_{\max }}-d_{\mathrm{cen}}\left(\boldsymbol{x}_{i}\right)}{d_{\mathrm{cen}_{\max }}}\right)
$$

where $h\left(\boldsymbol{x}_{i}\right)$ is the value in the Hough map corresponding to circle $i, r\left(\boldsymbol{x}_{i}\right)$ is the radius of this circle, $d_{\text {cen }}\left(\boldsymbol{x}_{i}\right)$ is the Euclidean distance of the circle center to the slice center, and $d_{\text {cen }}$ max is half the side length of the oblique square 2-D slice. It is sufficient to only check the first $n=10$ maxima found in the Hough map.

Adhering to the adaptive model, we make sure to construct only slices approximately orthogonal to the running direction of the pulmonary artery and hence always showing its roughly circular shape required for the Hough transform.

\subsubsection{NURBS Fitting}

In a least squares sense, we approximate three NURBS curves (each having five control points) to the centerline points of the trunk, left, and right pulmonary artery. The control point at the branching is identical in its position and tangent to the last control point of the pulmonary trunk and the first control point of the left and right pulmonary artery, so we get a smooth transition between the NURBS curves.

The three NURBS curves are fitted to a likelihood image of the centerline, which is created by performing a morphological opening on the input image, computing edge voxels by gradient magnitude and variance filtering, and applying a Euclidean distance transform to the edge image. As already detailed in our previous works, ${ }^{5,11}$ for likelihood image generation we first perform a morphological opening using a sphere of size $r_{\mathrm{PA}}$ on the median-filtered image to reduce variance of voxel intensities. Next, we detect edges in the opened image by computing the gradient magnitude and only leave voxels with a magnitude greater than $t_{\Delta_{\mathrm{E}}}$. As the variance of intensities inside blood vessels is usually low, we can reduce false edge candidates by computing the standard deviation at each edge candidate within a sphere of radius $r_{\sigma_{\mathrm{E}}}$ in the opened image and only leaving candidates whose standard deviation is greater than $t_{\sigma_{\mathrm{E}}}$. Last, to generate the final likelihood image we apply a Euclidean distance transform to the edge image supplemented with "artificial" edge voxels obtained from all voxels of the aortic arch segmentation performed during preprocessing. The likelihood image shows the distance between a voxel and its nearest edge voxel and thus the likelihood of a voxel to be part of the centerline. Using additional "artificial" edge voxels we ensure that all aortic arch voxels have zero likelihood to be part of the pulmonary artery.

In contrast enhanced data, the intensity distribution varies a lot within the contrasted region, leading to unwanted edges inside the pulmonary artery. To only consider its wall, we compute the average image intensity at the initial centerline points. If the average intensity exceeds a threshold $t_{\mathrm{C}}$, we adjust all input voxels to be smaller or equal to $t_{\mathrm{C}}$ before performing above likelihood image generation steps. 
For the final fitting process of the NURBS curve to the likelihood image, we use the Powell optimizer to maximize following expression:

$$
\underset{\boldsymbol{P}_{i}}{\arg \max }(\sum_{c=1}^{3} \sum_{j=1}^{m_{c}} \overbrace{d_{\mathrm{L}}^{2}\left(N_{c}\left(\frac{j}{m_{c}}\right)\right)}^{\text {likelihood term }}-\overbrace{\max ^{2}\left(0, r_{\min }-r\left(N_{c}\left(\frac{j}{m_{c}}\right)\right)\right)}^{\text {penalty term }})
$$

Here, $d_{\mathrm{L}}(\boldsymbol{X})$ is the Euclidean distance value of voxel $\boldsymbol{X}$ in the likelihood image, $N_{c}(u)=\sum_{i=1}^{k} R_{i, p_{c}} \boldsymbol{P}_{i_{c}}$ is one of the three NURBS curves with $R_{i, p_{c}}$ being its rational basis functions (of degree $p=3$ in our case) and $\boldsymbol{P}_{i_{c}}=\left(x_{i_{c}}, y_{i_{c}}, z_{i_{c}}\right)^{T}$ its $i=1 \ldots k$ control points, $m_{c}$ is the number of sampling points along the curve (we sample every millimeter), and $r\left(N_{c}(u)\right)=\frac{\left\|N_{c}^{\prime}(u)\right\|^{3}}{\left\|N_{c}^{\prime}(u) \times N_{c}^{\prime \prime}(u)\right\|}$ is the radius of curvature at $u$.

In contrast to our previous work, ${ }^{5,11}$ we here introduce a penalty term to penalize very high curvatures, i.e. penalize high bending (note that curvature is the reciprocal of the radius of curvature), with $r_{\min }$ being the minimum pulmonary artery radius found during Hough transform, as it is impossible for the pulmonary artery to have a smaller radius of curvature than its own radius. Compared to previous work, ${ }^{2,11,15}$ where one or more models need to be fitted globally as well as locally using several energy terms, we here greatly reduce the amount and complexity of optimizations to a single local minimization, making the approach faster and stable.

Finally, we recover the lumen of the pulmonary artery by a reverse Euclidean distance transform along all centerline voxels, followed by a procedure to deal with false edges. ${ }^{5,11}$ In detail, we initialize each centerline voxel with the corresponding Euclidean distance from the likelihood image and draw a sphere of radius equal to this distance. As the likelihood image may still contain wrong edges, we grow each sphere iteratively, until the standard deviation of all voxels within the sphere exceeds $t_{\sigma_{R}}$. Each iteration increases the radius of the sphere by the smallest of the three spatial resolutions of the input image.

\section{RESULTS}

We applied our method to 10 contrast enhanced and 30 non-contrast 3-D chest CT data sets (randomly selected from our database) of various hospitals, scanners, and acquisition parameters. The data sets consist of $99-838$ slices spaced $0.4-1.5 \mathrm{~mm}$. Each axial slice has $512 \times 512$ pixels of size $0.5-0.665 \mathrm{~mm}$. In all 40 cases, the centerline and lumen of the pulmonary artery could be extracted successfully.

Table 1: Values of parameters used for evaluation (HU refers to Hounsfield units).

\begin{tabular}{rrl|rrr|rrr|rrl}
$s_{\mathrm{m}}:$ & $3 \times 3 \times 3$ & voxels & $t_{\mathrm{H}_{\min }}:$ & -60 & $\mathrm{HU}$ & $v_{\mathrm{H}}:$ & 5 & pixels & $t_{\Delta_{\mathrm{E}}}:$ & 10 & $\mathrm{HU}$ \\
\hline$t_{\mathrm{C}}:$ & 100 & $\mathrm{HU}$ & $t_{\mathrm{H}_{\max }}:$ & 40 & $\mathrm{HU}$ & $\sigma_{\mathrm{H}}:$ & 5 & pixels & $r_{\sigma_{\mathrm{E}}}:$ & 3 & voxels \\
\hline$r_{\mathrm{PA}}:$ & 4 & $\mathrm{~mm}$ & $r_{\mathrm{H}_{\min }}:$ & 5 & $\mathrm{~mm}$ & & & & $t_{\sigma_{\mathrm{E}}}:$ & 15 & $\mathrm{HU}$ \\
\hline & & & $r_{\mathrm{H}_{\max }}:$ & 18 & $\mathrm{~mm}$ & & & & $t_{\sigma_{\mathrm{R}}}:$ & 8 & $\mathrm{HU}$
\end{tabular}

We set all parameters according to our previous $\operatorname{work}^{5}$ (see Table 1). However, the threshold for contrast material $\left(t_{\mathrm{C}}\right)$ was reduced from $200 \mathrm{HU}$ to $100 \mathrm{HU}$, as in contrast enhanced $\mathrm{CT}$ the pulmonary artery voxels are usually of much lower intensity than the aortic arch voxels, and the search region for the Hough circle radii $\left[r_{\mathrm{H}_{\min }}, r_{\mathrm{H}_{\max }}\right]$ was reduced from $[7,28] \mathrm{mm}$ to $[5,18] \mathrm{mm}$, as the pulmonary artery is thinner than the aortic arch.

To quantify our results, we also manually segmented the pulmonary artery for the first 25 data sets (10 contrast enhanced and 15 non-contrast), which took approximately one hour per data set.* We compared the 3 -D volume overlap of the automatic results and the manual segmentations, resulting in an average Jaccard index of $87 \%$, sensitivity of $93 \%$, and specificity of $98 \%$ (considering voxels within the bounding box of the two segmentations only). The mean minimum distance between the boundaries of the manual and automatic lumen segmentation was $0.5 \mathrm{~mm}$. All resuls are detailed in Table 2 and exemplified in Fig. 2 and 3.

Depending on the size of a data set, a complete segmentation including preprocessing (i.e. aortic arch segmentation) takes about 5 minutes on a workstation with two 64-bit Quad-Core Intel Xeon 5355 processors.

${ }^{*}$ Due to limited time we omitted the quantitative evaluation of the remaining 15 results, as a thorough visual inspection did not reveal any significant qualitative differences to the first 25 results. 
Table 2: Quantitative results.

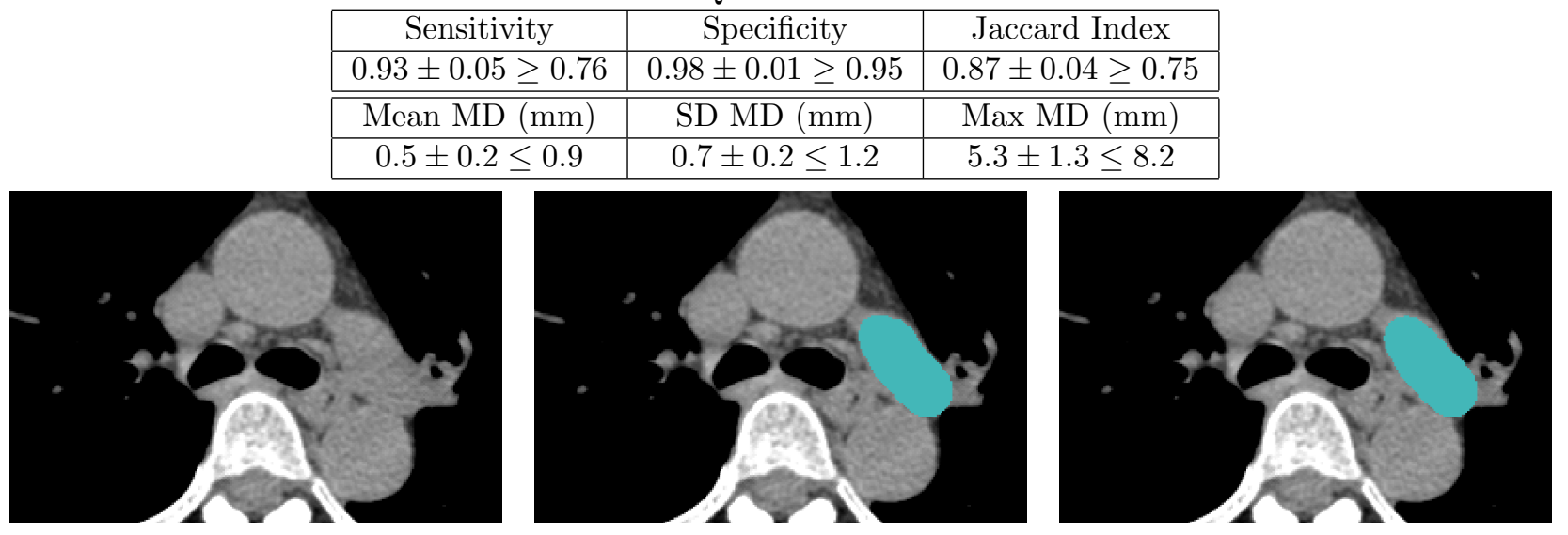

(a) Original image.

(b) Manual segmentation.

(c) Automatic segmentation.

Figure 2: Exemplary extraction result shown in an axial slice containing superior vena cava, ascending and descending aorta, pulmonary artery, left and right main bronchus, and esophagus.

\section{DISCUSSION}

Currently our model slices are using a fixed inter-slice distance of $5 \mathrm{~mm}$. While this value provides an overall good result for our 40 evaluation data sets, it should be automatically adjusted to expand over the real size of the mediastinum, which is varying for each patient. This could be achieved by roughly estimating the position of the right ventricle and the mediastinal surface of the left and right lungs and will be part of our future work.

As the pulmonary artery is not always circular due to pressure from surrounding vasculature or the bronchial tree and in particular where the pulmonary trunk begins at the base of the right ventricle, our recovery method sometimes could not fully extract the lumen of the pulmonary artery by the reverse Euclidean transform (cf. Fig. 5 and 6). This will be a challenging task in future work, as cardiac motion artifacts and merging tissue make a full recovery difficult, even when using deformable models or the like, which can also leak into surrounding adjacent tissue.

However, as can be seen from the quantitative analysis and the exemplary results shown in Fig. 2-6, the overall shape of the pulmonary artery was recovered well. Table 3 gives a comparison of our method to the current state of the art in pulmonary artery segmentation.

\section{CONCLUSIONS}

We presented a new method for the automatic extraction of the pulmonary artery in 3-D chest CT based on an adaptive model, circular Hough and Euclidean transform, and spline fitting. We successfully applied the method to a large number of cases. It works robust on a large number of non-contrast and contrast enhanced data sets and improves the current state of the art. ${ }^{2,11-14}$

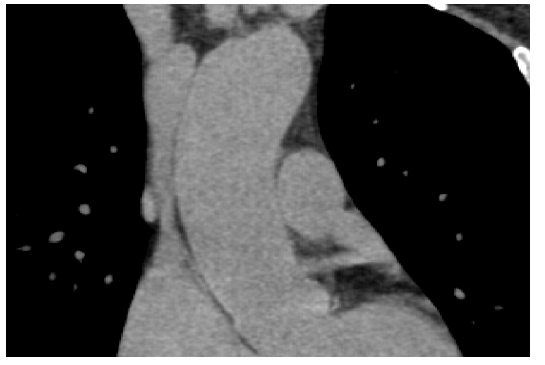

(a) Original image.

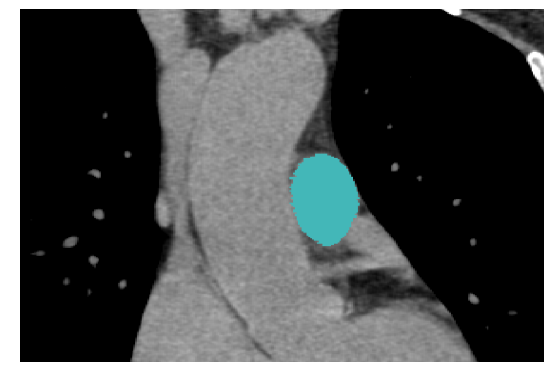

(b) Manual segmentation.

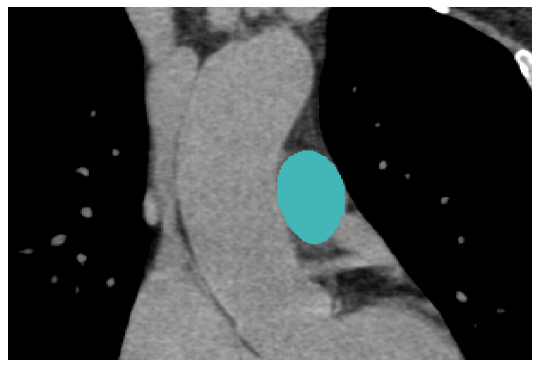

(c) Automatic segmentation.

Figure 3: Exemplary extraction result shown in a coronal slice containing aortic arch and pulmonary artery. 


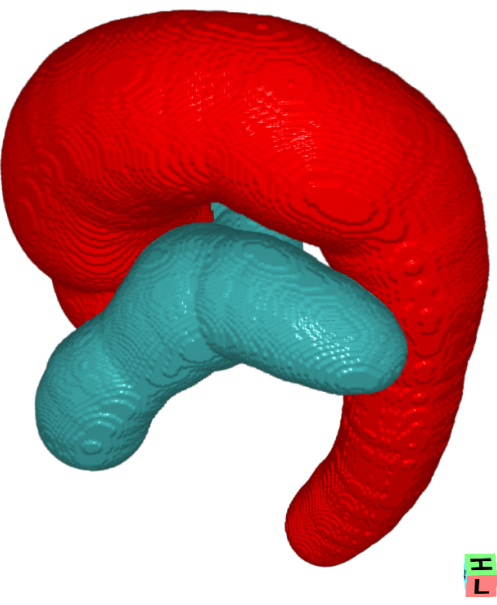

I

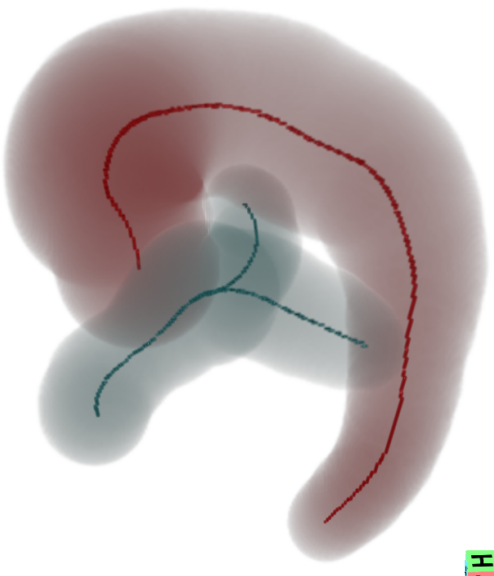

I

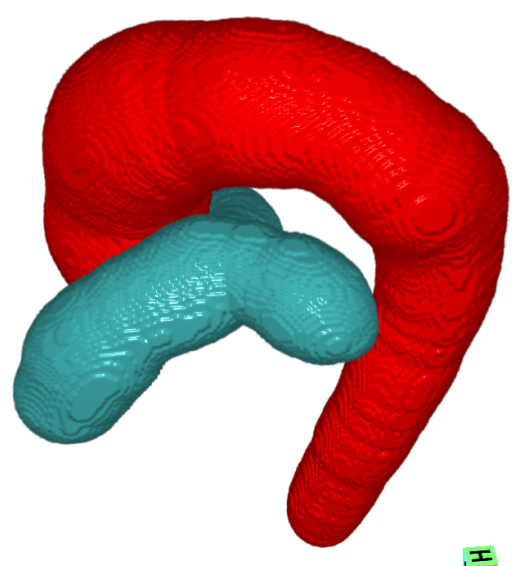

I

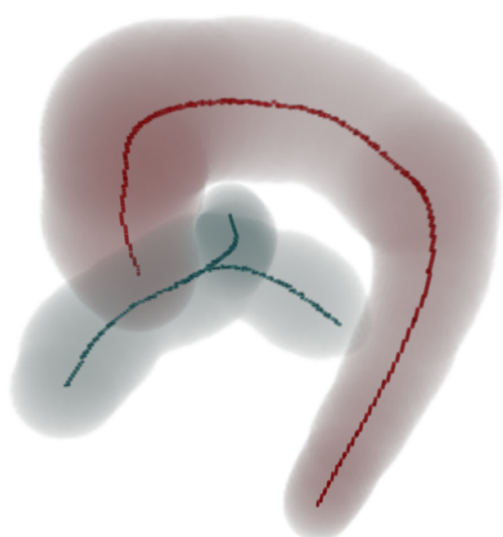

Ix

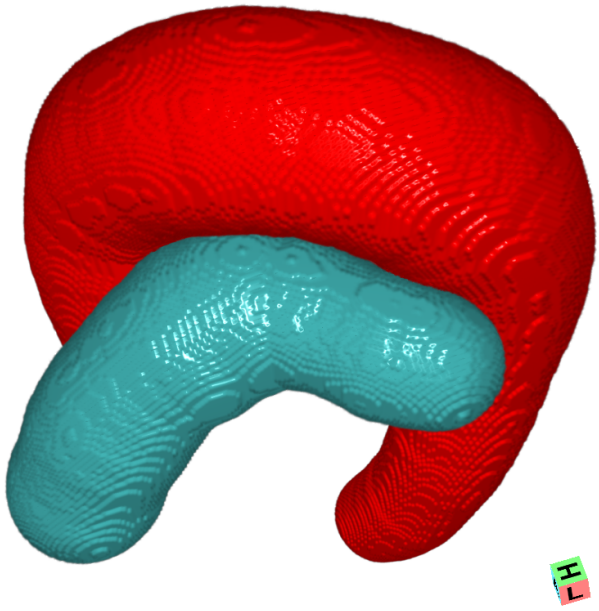

采

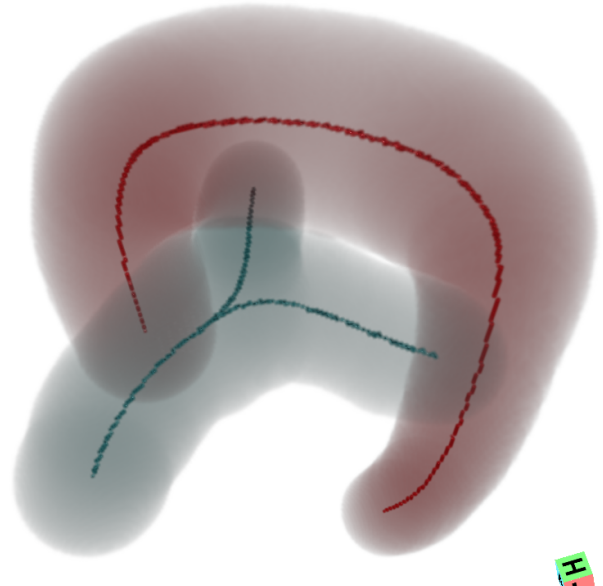

F

Figure 4: Exemplary final extraction results, rendered (top) opaque and (bottom) transparent also showing the centerline.

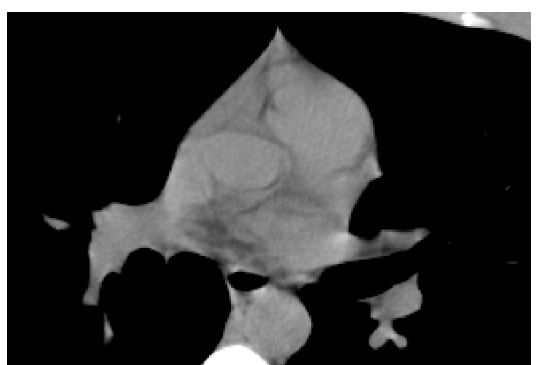

(a) Original image.

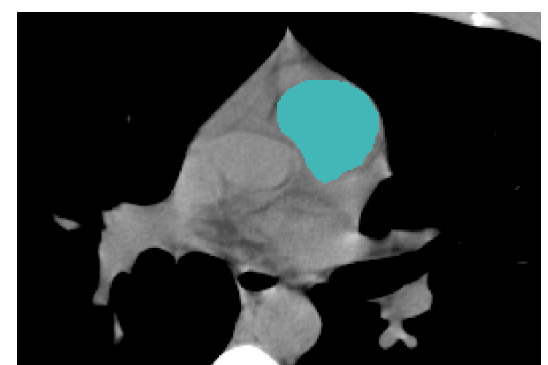

(b) Manual segmentation.

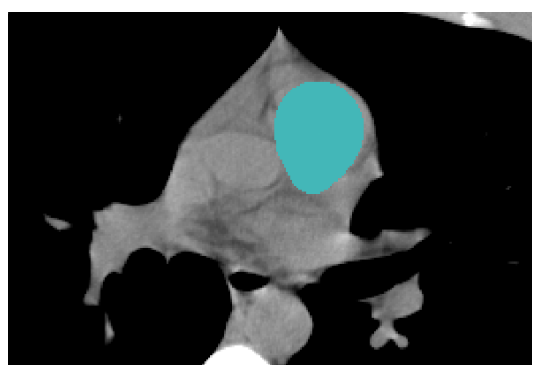

(c) Automatic segmentation.

Figure 5: Exemplary problematic extraction result, where we could not accurately recover the lumen of the pulmonary trunk close to the right ventricle due to imaging artifacts and insufficient roundness of the pulmonary artery. An axial slice containing ascending and descending aorta, pulmonary artery, left and right main bronchus, and esophagus is shown. 


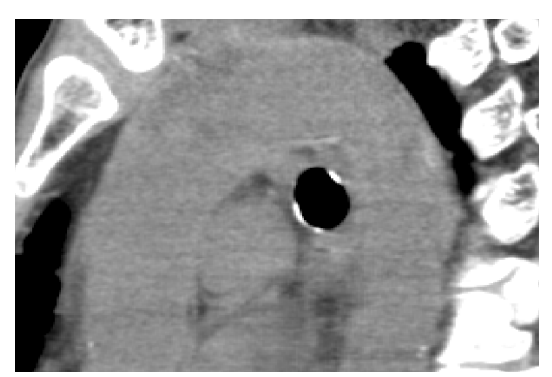

(a) Original image.

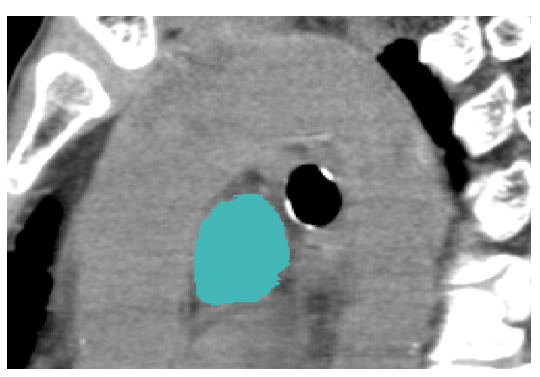

(b) Manual segmentation.

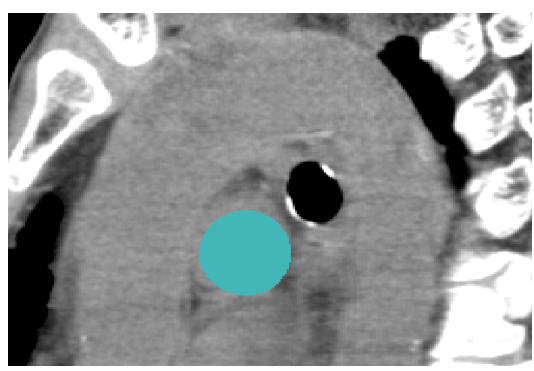

(c) Automatic segmentation.

Figure 6: Exemplary problematic extraction result, where we could not fully recover the lumen of the pulmonary artery due to imaging artifacts and insufficient roundness of the pulmonary artery. A sagittal slice containing aortic arch, pulmonary artery, and left main bronchus is shown.

Table 3: Comparison of our method to the state of the art in automatic pulmonary artery extraction.

\begin{tabular}{c|c|c|c|c} 
Method & Manual Seeds & Non-/Contrast & Successful/Cases & Mean MD $(\mathrm{mm})$ \\
\hline \hline Sebbe et al. ${ }^{12}$ & $5-8$ & No/Yes & $6 / 6$ & NA \\
\hline Linguraru et al. $^{13}$ & 1 & No/Yes & $8 / 8$ & NA \\
\hline Vitanovski et al. $^{14}$ & 0 & No/Yes & $50 / 50$ & $2.2 \pm 2.1$ \\
\hline Kitasaka et al. $^{11}$ & 0 & Yes/No & $7 / 7$ & $0.9 \pm 0.4$ \\
\hline Taeprasartsit \& Higgins $^{2}$ & 0 & Yes/Yes & $20 / 25$ & NA \\
\hline Ours & 0 & Yes/Yes & $40 / 40$ & $0.5 \pm 0.2$
\end{tabular}

\section{ACKNOWLEDGMENTS}

This work was supported in part by the JSPS postdoctoral fellowship program for foreign researchers, by the program of formation of innovation center for fusion of advanced technologies "Establishment of early preventing medical treatment based on medical-engineering for analysis and diagnosis" funded by MEXT, by a Grant-in-Aid for Science Research funded by JSPS, and by a Grant-in-Aid for Cancer Research funded by the Ministry of Health, Labour and Welfare, Japan.

\section{REFERENCES}

[1] Rusch, V. W., Asamura, H., Watanabe, H., Giroux, D. J., Rami-Porta, R., Goldstraw, P., and the Members of the IASLC Staging Committee, "The IASLC lung cancer staging project: A proposal for a new international lymph node map in the forthcoming seventh edition of the TNM classification for lung cancer," Journal of Thoracic Oncology 4, 568-577 (May 2009).

[2] Taeprasartsit, P. and Higgins, W. E., "System for definition of the central-chest vasculature," in [SPIE Medical Imaging], (2009).

[3] Sihoe, A. D. L. and Yim, A. P. C., "Lung cancer staging," Journal of Surgical Research 117, 92-106 (2004).

[4] Feuerstein, M., Kitasaka, T., and Mori, K., "Adaptive branch tracing and image sharpening for airway tree extraction in 3-D chest CT," in [Second International Workshop on Pulmonary Image Analysis], (2009).

[5] Feuerstein, M., Kitasaka, T., and Mori, K., "Automated anatomical likelihood driven extraction and branching detection of aortic arch in 3-D chest CT," in [Second International Workshop on Pulmonary Image Analysis], (2009).

[6] Masutani, Y., MacMahon, H., and Doi, K., "Computerized detection of pulmonary embolism in spiral CT angiography based on volumetric image analysis," IEEE Transactions on Medical Imaging 21(12), 15171523 (2002).

[7] Shikata, H., Hoffman, E. A., and Sonka, M., "Automated segmentation of pulmonary vascular tree from 3D CT images," in [SPIE Medical Imaging], (2004).

[8] Agam, G., Samuel G. Armato, I., and Wu, C., "Vessel tree reconstruction in thoracic CT scans with application to nodule detection," IEEE Transactions on Medical Imaging 24(4), 486-499 (2005). 
[9] Zhou, C., Chan, H.-P., Sahiner, B., Hadjiiski, L. M., Chughtai, A., Patel, S., Wei, J., Ge, J., Cascade, P. N., and Kazerooni, E. A., "Automatic multiscale enhancement and segmentation of pulmonary vessels in CT pulmonary angiography images for CAD applications," Medical Physics 34(12), 4567-4577 (2007).

[10] Kaftan, J. N., Kiraly, A. P., Bakai, A., Das, M., Novak, C. L., and Aach, T., "Fuzzy pulmonary vessel segmentation in contrast enhanced CT data," in [SPIE Medical Imaging], (2008).

[11] Kitasaka, T., Mori, K., Hasegawa, J., Toriwaki, J., and Katada, K., "Automated extraction of aorta and pulmonary artery in mediastinum from 3D chest X-ray CT images without contrast medium," in [SPIE Medical Imaging], (2002).

[12] Sebbe, R., Gosselin, B., Coche, E., and Macq, B., "Segmentation of opacified thorax vessels using modeldriven active contour," in [IEEE Engineering in Medicine and Biology], 2535-2538 (2005).

[13] Linguraru, M. G., Mukherjee, N., Uitert, R. L. V., Summers, R. M., Gladwin, M. T., Machado, R. F., and Wood, B. J., "Pulmonary artery segmentation and quantification in sickle cell associated pulmonary hypertension," in [SPIE Medical Imaging], (2008).

[14] Vitanovski, D., Ionasec, R. I., Georgescu, B., Huber, M., Taylor, A. M., Hornegger, J., and Comaniciu, D., "Personalized pulmonary trunk modeling for intervention planning and valve assessment estimated from CT data," in [Medical Image Computing and Computer Assisted Intervention], (2009).

[15] Taeprasartsit, P. and Higgins, W. E., "Method for extracting the aorta from 3D CT images," in [SPIE Medical Imaging], (2007).

[16] Kunz, P., Scholz, M., Haas, B., and Coradi, T., "Anatomic orientation in medical images," (2008). 